\title{
Synthesis of ferrofluid based nanoarchitectured polypyrrole composites and its application for electromagnetic shielding
}

\author{
Swati Varshney ${ }^{\mathrm{a} b, \mathrm{c}}$, Anil Ohlan ${ }^{\mathrm{d}}$, V.K. Jain ${ }^{\mathrm{c}}$, V.P. Dutta ${ }^{\mathrm{b}}$, S.K. Dhawan ${ }^{\mathrm{a}, *}$ \\ ${ }^{a}$ Polymeric \& Soft Materials Section, National Physical Laboratory (CSIR), New Delhi 110012, India \\ ${ }^{\mathrm{b}}$ Department of Chemistry, Delhi Institute of Tool Engineering, Okhla, New Delhi 110020, India \\ ${ }^{\mathrm{c}}$ Amity Institute of Advanced Research and Studies, Materials and Devices, AIARS (MED), Amity University, Noida, UP 201303, India \\ ${ }^{\mathrm{d}}$ Department of Physics, M.D. University, Rohtak, Haryana 124001, India
}

\section{H I G H L I G H T S}

- Aqueous ferrofluid has been incorporated in polypyrrole matrix leads to PFF nanocomposites.

- PFF composites shows conductivity of the order of $10^{-2} \mathrm{~S} \mathrm{~cm}^{-1} \&$ saturation magnetization of $5.5 \mathrm{emu}^{-1}$.

- Shielding effectiveness of $23.5 \mathrm{~dB}$ $\left(\mathrm{SE}_{\mathrm{A}} \sim 20.4 \mathrm{~dB} \& \mathrm{SE}_{\mathrm{R}} \sim 3.1 \mathrm{~dB}\right)$ has been achieved.

- Shielding effectiveness depends on the ferrofluid loading.

\section{A R T I C L E I N F O}

\section{Article history:}

Received 17 June 2013

Received in revised form

2 October 2013

Accepted 12 October 2013

\section{Keywords:}

Composite materials

Nanostructures

Electron microscopy

Chemical synthesis

Dielectric properties
G R A P H I C A L A B S T R A C T

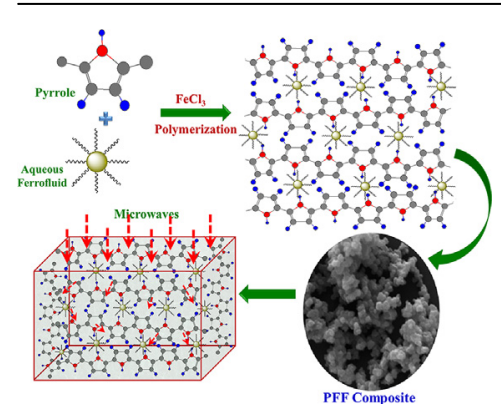

\begin{abstract}
A B S T R A C T
The monodispersion of magnetic nanoparticles in conducting polymer is the prerequisite to make a high quality composite for tunable electromagnetic interference (EMI) shielding. To meet this challenge, we have designed and synthesized ferrofluid based nanoarchitectured polypyrrole composites containing $\mathrm{Fe}_{3} \mathrm{O}_{4}(8-12 \mathrm{~nm})$ via in situ oxidative polymerization. To tune the microwave signals, polypyrrole composites (PFF) with different monomer/ferrofluid weight ratios have been prepared and characterized in microwave frequency domain. A maximum shielding effectiveness value of $\mathrm{SE}_{\mathrm{A}(\max )}=20.4 \mathrm{~dB}$ ( $\sim 99 \%$ attenuation) due to the absorption of microwave has been observed in the frequency range of $12.4-18 \mathrm{GHz}$ and attenuation level varied with ferrofluid loading. The electrical conductivity of PFF composite is of the order of $10^{-2} \mathrm{~S} \mathrm{~cm}^{-1}$ order and having superparamagnetic

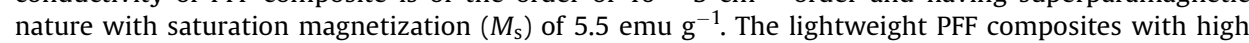
attenuations can provide full control over the atomic structure and are favorable for the practical EMI shielding application for commercial electronic appliances.
\end{abstract}

(C) 2013 Elsevier B.V. All rights reserved.

\section{Introduction}

The growths and development made in nano sciences and technologies have leaded high-density electronic devices operating at high frequency range, creating increased vulnerability to

\footnotetext{
* Corresponding author. Tel.: +91 11 45609401; fax: +91 1125726938

E-mail address: skdhawan@mail.nplindia.ernet.in (S.K. Dhawan).
}

electromagnetic interference (EMI). The electrical components of all types are subject to the interference induced by electric and magnetic signals. Therefore, in the present electronic era, all electronic devices and radiation sources requires electromagnetic shielding [1]. As a result, the demand for the microwave absorbers and electromagnetic shields in high frequency range is increasing day by day. All electromagnetic waves consist of two essential components, a magnetic field $(H)$ and electric field $(E)$ perpendicular to each other. The direction of wave propagation is at right 
angles to the plane containing the two components. The shields should have high conductance, thus metal coated or metal plated polymers are the most widely used materials for EMI shielding [25]. However, metal shields have poor mechanical flexibility, high weight, tendency to corrosion, and limited tuning of the SE, whereas polymers offer lightness, low cost, easy shaping, etc. [6,7]. Furthermore, the high filler loading increases the composite cost and thus limits its commercial use. Interest centered on the prospect of replacing the existing metal with conducting polymer to create a new field of application or to make existing ones less expensive. Compositing conducting/insulating polymers with inorganic filler like CNT, magnetic or metal nanoparticles are an excellent alternate to make a tunable shield in microwave regime [8-10]. A number of articles reported the synthesis and electrical properties of polymer nanocomposites of polypyrrole and polyaniline incorporating ferrite particles in the polymer matrix [1115]. Yang et al. reported a CNT polystyrene foam structure composite with EMI shielding effectiveness SE of $19 \mathrm{~dB}$ [16]. Wan et al. have reported the chemical method to prepare polypyrrole composite exhibiting magnetic and conducting properties [11]. Structural and physicochemical properties of composite materials are associated with the average particle size and its distribution, particle shape, surface characteristics, and the presence or absence of bonded molecules $[17,18]$.

Nanoscale ferrimagnetic fillers are the most attractive due to the intriguing properties arising from the nanosize and large surface area. The insertion of ferromagnetic nanoparticles may improve the magnetic and dielectric properties of host materials. Therefore, conjugated polymers combined with magnetic nanoparticles to form ferromagnetic nanocomposites provide an exciting system to investigate the possibility of exhibiting novel functionality to provide the better microwave absorption properties [19-21]. Recently, Dhawan et al. have investigated by the microwave absorption properties of the $\gamma-\mathrm{Fe}_{2} \mathrm{O}_{3}$ incorporated polyaniline, PEDOT nanocomposites with different loading [22,23]. The properties of these polymer composites depend on their micro/nanostructures and morphologies which is controlled by the synthesis method. Xie et al. has reported the $\mathrm{La} / \mathrm{Nd}$-doped barium-ferrite/polypyrrole nanocomposites by in situ chemical polymerization exhibiting magnetic and conducting properties [15]. However, the issue related to the homogeneous dispersion of nanoparticles in the polymer matrix is a great challenge, besides this selection of absorber based on lightweight, compatible with other packaging components and the cost of the fabrication process for commercial applications is another issue.

In this paper, we report a facile approach to design lightweight nano engineered polypyrrole composites with aqueous ferrofluid containing $\mathrm{Fe}_{3} \mathrm{O}_{4}(8-12 \mathrm{~nm})$ nanoparticles. The ferrofluid-based polypyrrole composite offers great potential for electromagnetic absorber due to geometrical effect and the existence of synergistic properties required to get efficient EMI shielding effectiveness. We investigated the structural, morphological and physical properties of ferrofluid based polypyrrole composite and tailored the microwave absorption properties in $12.4-18 \mathrm{GHz}$ frequency range by varying the concentration of ferrofluid.

\section{Experimental section}

\subsection{Material}

Pyrrole (Py), isopropyl alcohol, $\mathrm{FeCl}_{3} \cdot 6 \mathrm{H}_{2} \mathrm{O}, \mathrm{FeCl}_{2} \cdot 4 \mathrm{H}_{2} \mathrm{O}$ and aqueous ammonia solution, tetramethylammonium hydroxide (TMAH) purchased from Merck, India. All chemicals were of analytical grade and used as such. Monomer pyrrole has been distilled before use and double distilled water has been used throughout the experiment.

\subsection{Synthesis of the aqueous ferrofluid}

Water based ferrofluid (FF) has been prepared by coprecipitation technique. $4: 1 \mathrm{M}$ ratio of $1.0 \mathrm{M} \mathrm{FeCl}_{3}$ and $2.0 \mathrm{M}$ $\mathrm{FeCl}_{2}$ have been mixed together with $2 \mathrm{M} \mathrm{HCl}$ and co-precipitated by drop wise addition of $1 \mathrm{M}$ ammonium hydroxide solution at room temperature. The $\mathrm{pH}$ of the solution was maintained at 11-12. The resulting brownish black precipitate has been filtered out and washed with distilled water, followed by the addition of $25 \%$ tetramethylammonium hydroxide (TMAH) and stirred with a glass rod to suspend the nanoparticles in the carrier solvent. The homogeneously suspended nanoparticles so formed, show spikes when exposed to a strong magnet (Fig. 1), confirming the formation of magnetic ferrofluid.

\subsection{Synthesis of PFF nanocomposites}

The synthesis of polypyrrole ferrofluid composites has performed by in situ incorporation of aqueous ferrofluid in polypyrrole matrix to form mono dispersed nano magnets in the composites. Freshly prepared ferrofluid was charged to $1 \mathrm{M}$ pyrrole (Py) solution and kept homogenized for $1 \mathrm{~h}$ at 10,000 rpm using ultra homogenizer at room temperature. The resulted solution has been polymerized by drop wise addition of oxidant $\mathrm{FeCl}_{3}(0.2 \mathrm{M})$ at room temperature and stirred for $4-5 \mathrm{~h}$. The precipitates so obtained have been filtered out and washed with distilled water several times to remove the traces of unreacted $\mathrm{FeCl}_{3}$ and dried at $60{ }^{\circ} \mathrm{C}$ in vacuum oven for $36 \mathrm{~h}$. The dried precipitates have been grounded to powder samples of polypyrrole coated ferrofluid. For comparative study, polypyrrole (PPy) without ferrofluid along with several compositions of the PFF nanocomposites having different weight ratio of Py:FF::1:1 (PFF11), 1:2 (PFF12), 1:3 (PFF13) have also been synthesized by the same procedure as described above in order to check the effect of loading ferrofluid constituents in the polymer matrix on microwave absorption properties.

\section{Characterization}

The particle size and the morphology of ferrofluid and PFF composites have been examined by using high-resolution transmission electron microscopy (HRTEM, Technai G20-stwin) operated at an accelerating voltage of $200 \mathrm{kV}$ having a point resolution of $1.44 \AA$ and a line resolution of $2.32 \AA$ and scanning electron

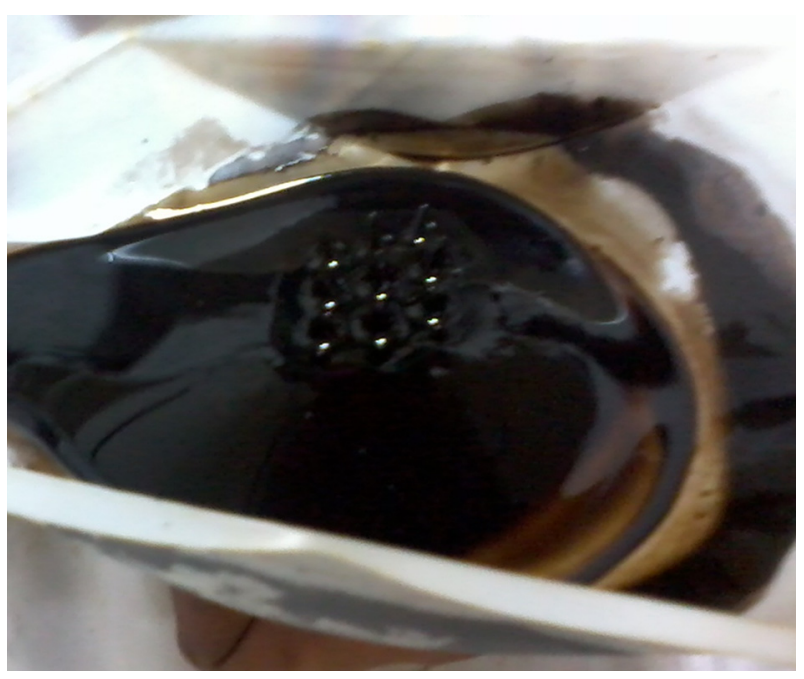

Fig. 1. Aqueous ferrofluid showing the spikes in the presence of a magnet. 
microscope (SEM LEO 440). Room temperature conductivity has been measured via four-probe method. Four contacts have been made on the compressed pellet $\left(13 \times 7 \mathrm{~mm}^{2}\right)$ of the composite samples using conducting silver paste and connected to the Keithley programmable current source (model 6221) and nanovoltmeter (model 2182A) and conductivity has been calculated based on ohm's law. The presence of ferrofluid in the polymer composites has been confirmed by X-ray diffraction (XRD) studies carried out on D8 Advance X-ray diffractometer (Bruker) using $\mathrm{Cu}$ $\mathrm{K} \alpha$ radiation $(\lambda=1.540598 \AA)$ in scattering range $(2 \theta)$ of $10-70^{\circ}$ with a scan rate of $0.02^{\circ} \mathrm{s}^{-1}$ and slit width of $0.1 \mathrm{~mm}$. The magnetic measurements of aqueous ferrofluid and PFF composites have been performed using the Vibrating sample magnetometer (VSM) (model 7304 Lakeshore cryotronics Inc. USA), having a maximum magnetic field of $1.2 \mathrm{~T}$ and the vibrating frequency of $76 \mathrm{~Hz}$. Electromagnetic shielding and dielectric measurements have been carried out on an Agilent E8362B Vector network analyzer in the frequency range of $12.4-18 \mathrm{GHz}$ (Ku-band). Powder samples have been compressed in the form of rectangular pellets $\left(15.8 \times 7.9 \times 2 \mathrm{~mm}^{3}\right)$, inserted in a copper sample holder, and connected between the waveguide flanges of a network analyzer.

\section{Results and discussion}

\subsection{Mechanism of PFF formation}

A ferrofluid is a collection of superparamagnetic nanoparticles that are suspended in a liquid as long as they do not aggregate. Tetramethylammonium hydroxide (TMAH) was used as a surfactant in the preparation of aqueous ferrofluid to stabilize the $\mathrm{Fe}_{3} \mathrm{O}_{4}$ nanoparticles in the colloidal suspension by encapsulating them within a shell. The surfactant provides the coating of magnetic nanoparticles in a micelle-like fashion which interact with the conducting polymer via hydrogen bonding. During polymerization, the addition of $\mathrm{FeCl}_{3}$ to the aqueous solution having pyrrole (monomer) and $\mathrm{Fe}_{3} \mathrm{O}_{4}$ particles lead to the oxidative polymerization in which pyrrole is oxidized and form pyrrole cationic free radicals. These pyrrole cationic free radicals have been adsorbed on the surface of the $\mathrm{Fe}_{3} \mathrm{O}_{4}$ and polymerized continuously which subsequently combine with another unit to form neutral dimer. Further oxidation of this dimer leads to the formation of a trimer, tetramer and finally to the polymer composite having the $\mathrm{Fe}_{3} \mathrm{O}_{4}$ nanoparticles embedded in between the polypyrrole chains. Schematic representation of incorporation of $\mathrm{FF}$ in polypyrrole matrix is shown in Fig. 2. The presence of FF in polymer matrix has been confirmed by the X-ray diffraction patterns of the composites. Due to the core shell type of morphology, it is observed that the dc conductivity at room temperature of these composites does not deteriorate to much extent after incorporation of ferrofluid as compared to the polypyrrole. The conductivity value of $0.11,0.08$ and $0.05 \mathrm{~S} \mathrm{~cm}^{-1}$ has been calculated for PFF11, PFF12 and PFF13 composites respectively. Whereas the polypyrrole shows the conductivity of $0.14 \mathrm{~S} \mathrm{~cm}^{-1}$ that reveals the decreases in conductivity with the concentration of ferrofluid due to hindrance in the conduction path.

\subsection{X-ray diffraction studies}

X-ray diffraction pattern of PFF nanocomposites and FF is shown in Fig. 3. The main peaks for FF have been observed at $2 \theta=30.18$ $(d=2.959), 35.55(d=2.524), 43.18(d=2.094), 57.14(d=1.611)$, and $62.71^{\circ}(d=1.480 \AA)$ corresponding to the (220), (311), (400), (511), and (440) reflections respectively, which matches with the standard XRD pattern of $\mathrm{Fe}_{3} \mathrm{O}_{4}$ (Powder Diffraction File, JCPDS No.

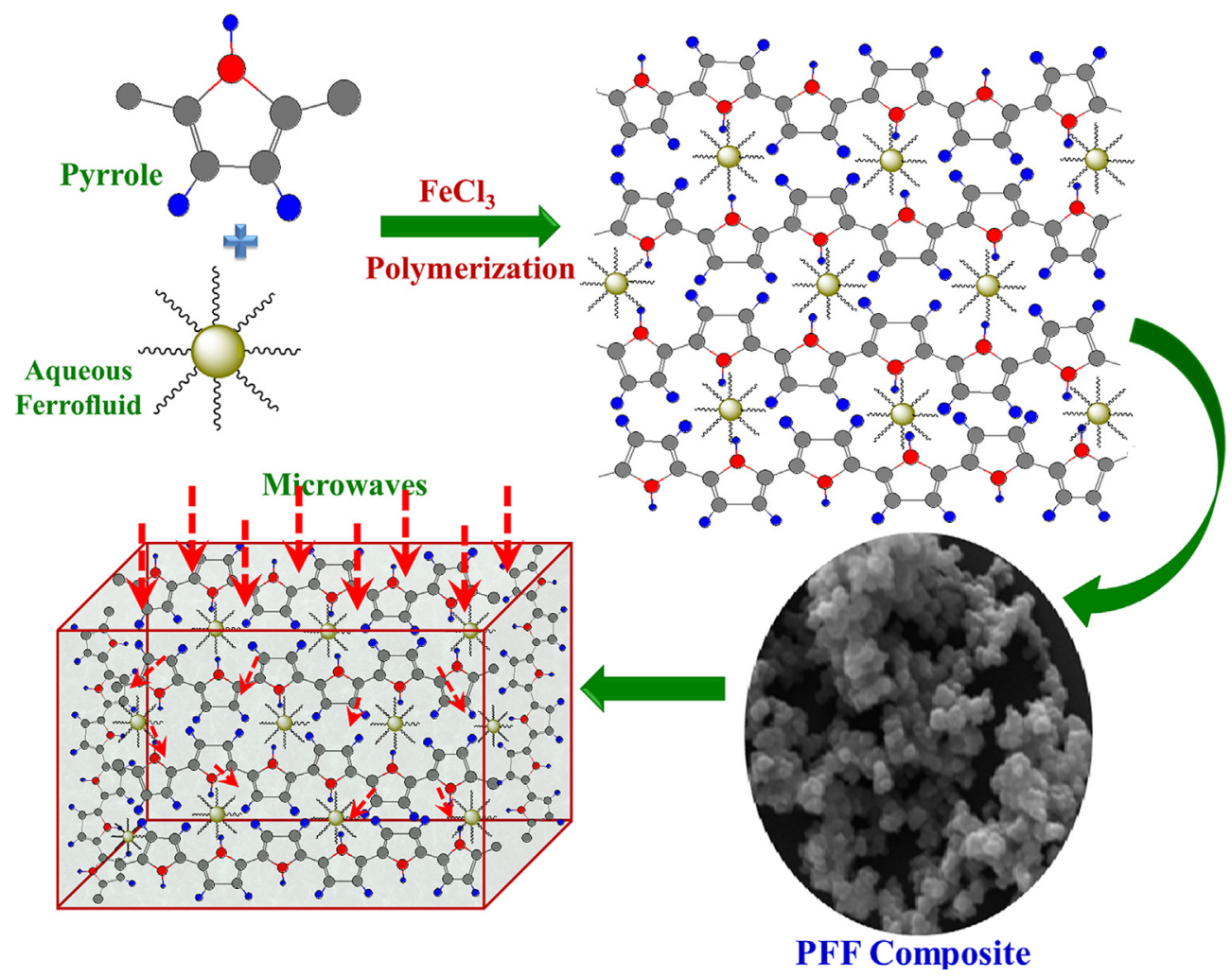

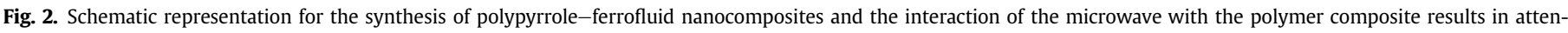
uation due to the scattering with the nanoparticles. 


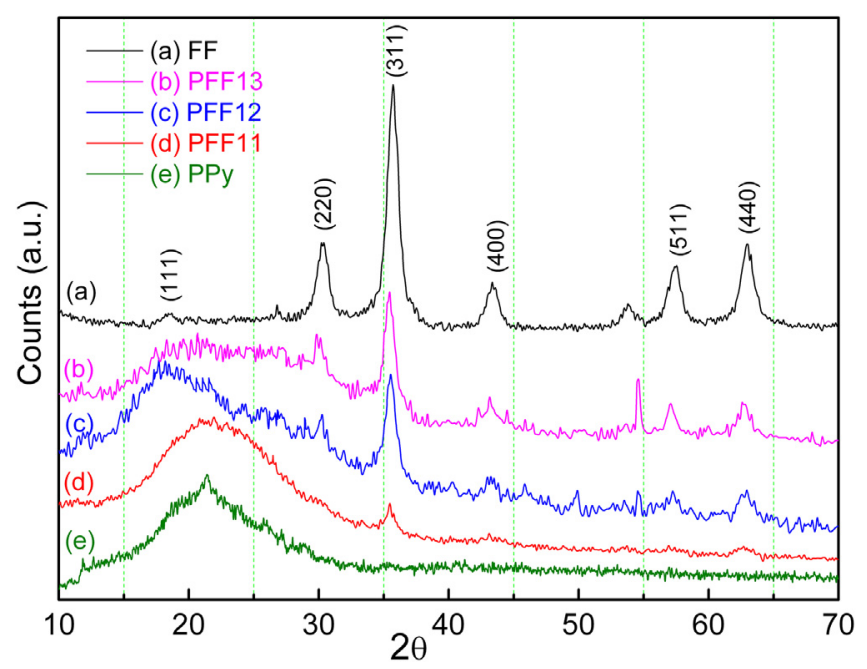

Fig. 3. X-ray diffraction plots of (a) FF, (b) PFF13, (c) PFF12, (d) PFF11, and (e) PPy. Plots (b), (c) and (d) confirm the presence of $\mathrm{Fe}_{3} \mathrm{O}_{4}$ in the polymer composites.

88-0315). The peaks present in FF have also been observed in the PFF composites which designate the presence of $\mathrm{Fe}_{3} \mathrm{O}_{4}$ phase in the polymer. While the presence of PPy and its amorphous nature is confirmed by the broad peak centered around at $2 \theta=21.4^{\circ}$ $(d=4.150 \AA$ ), corresponding to the scattering from polymer chains at the interplanar spacing of protonated polypyrrole [24,25]. It is also observed that the intensity of polypyrrole peak suppressed with the increase in concentration of more crystalline $\mathrm{Fe}_{3} \mathrm{O}_{4}$ in the composites. The crystallite size of $\mathrm{Fe}_{3} \mathrm{O}_{4}$ particle can be calculated by line broadening using Scherer's formula

$D=\frac{k \lambda}{\beta \cos \theta}$

where $\lambda$ is the wavelength $\mathrm{Cu} \mathrm{K} \alpha$ line, $k$ the shape factor, $D$ is the crystallite size for the individual peak of the crystal in angstroms, $\theta$ the Bragg angle in degrees, and $\beta$ is the line broadening measured by half-height in radians. The value of $k$ is often assigned a value of 0.89 , which depends on several factors, including the Miller index of the reflecting plane and the shape of the crystal. For $\mathrm{Fe}_{3} \mathrm{O}_{4}$ nanoparticles, the diffraction peak at $2 \theta=35.55^{\circ}$, showing the most strong intensity, is chosen to calculate the average size of the $\mathrm{Fe}_{3} \mathrm{O}_{4}$ nanoparticles and estimated as $8.7 \mathrm{~nm}$.

\subsection{Microscopy analysis}

Scanning electron micrographs (SEM) of polypyrrole composites are shown in Fig. 4. SEM of PFF11 sample has predominantly uniform nanospheres (Fig. 4a) and porous in nature whereas coral like morphology has been observed for PFF13 sample (Fig. 4b). It has been observed that the morphology of the micro/nanostructures is strongly affected by the structure of the monomer, dopant, and an oxidant, as well as by the preparation conditions. The preparation conditions include the concentrations of dopant, oxidant, monomer, and the molar ratio of the dopant and oxidant to monomer, as well as the reaction temperature, time, and stirring [26]. Interestingly, in the present system, these factors are same for all PFF composites but a change in morphology was observed due to variation in the concentration of ferrofluid in the feed system. In other words, the change in morphology of the PFF composites is mainly due ferrofluid-pyrrole complexes formed in the water phase.

Fig. 5 demonstrates the high-resolution TEM images of ferrofluid (a \& d), PFF11 (b \& e) and PFF13 (c \& f) nanocomposites. In

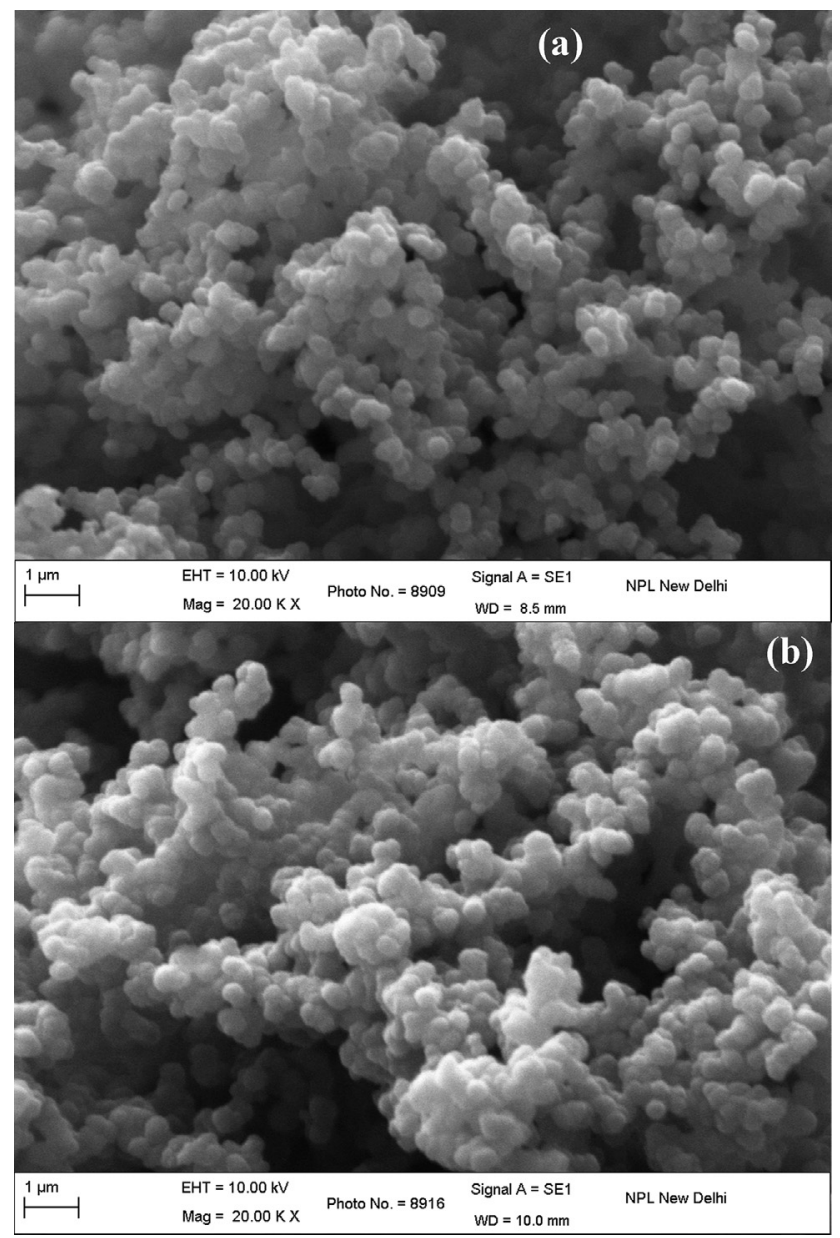

Fig. 4. Scanning electron microscope images of polypyrrole-ferrofluid nanocomposites (a) PFF11 and (b) PFF13.

Fig. 5(a) irregular shaped $\mathrm{Fe}_{3} \mathrm{O}_{4}$ nanoparticles with diameter of $8-$ $12 \mathrm{~nm}$ has been observed which is consistent with the calculated value based on XRD results. Some of them formed multi-particle aggregates owing to the magneto-dipole interactions between nanoparticles [27]. From Fig. 5(b) and (c) it is observed that PFF11 and PFF13 samples have well dispersed $\mathrm{Fe}_{3} \mathrm{O}_{4}$ nanoparticles in the polypyrrole matrix, look like a bunch of cotton balls with entangled black seeds. The presence of encapsulated magnetic and dielectric core into conducting crust is very helpful in improving the absorption of the EM wave. In Fig. 5(d)-(f), the conducting cover of the particles gives an impression of an amorphous behavior of polypyrrole as no fringes have been observed while the $d$-spacing of $0.25 \mathrm{~nm}$ (311 plane) reveals the presence of $\mathrm{Fe}_{3} \mathrm{O}_{4}$ nanoparticles in the polymer matrix.

\subsection{Magnetic measurements}

The magnetic properties of the ferrofluid and PFF composites have been analyzed using the $M-H$ curves (Fig. 6). The saturation magnetization $\left(M_{S}\right)$ value of the aqueous FF has been found to be $9.05 \mathrm{emu}^{-1}$ at an external field of $5 \mathrm{kOe}$ having a small value of coercivity and negligible retentivity with no hysteresis loop, indicating its superparamagnetic nature. When aqueous ferrofluid having $\mathrm{Fe}_{3} \mathrm{O}_{4}$ nanoparticles is incorporated in the PPy matrix in weight to volume ratio of 1:1 (PFF11), the saturation magnetization $\left(M_{\mathrm{S}}\right)$ value was found to be $1.3 \mathrm{emu} \mathrm{g}^{-1}$. However, on 

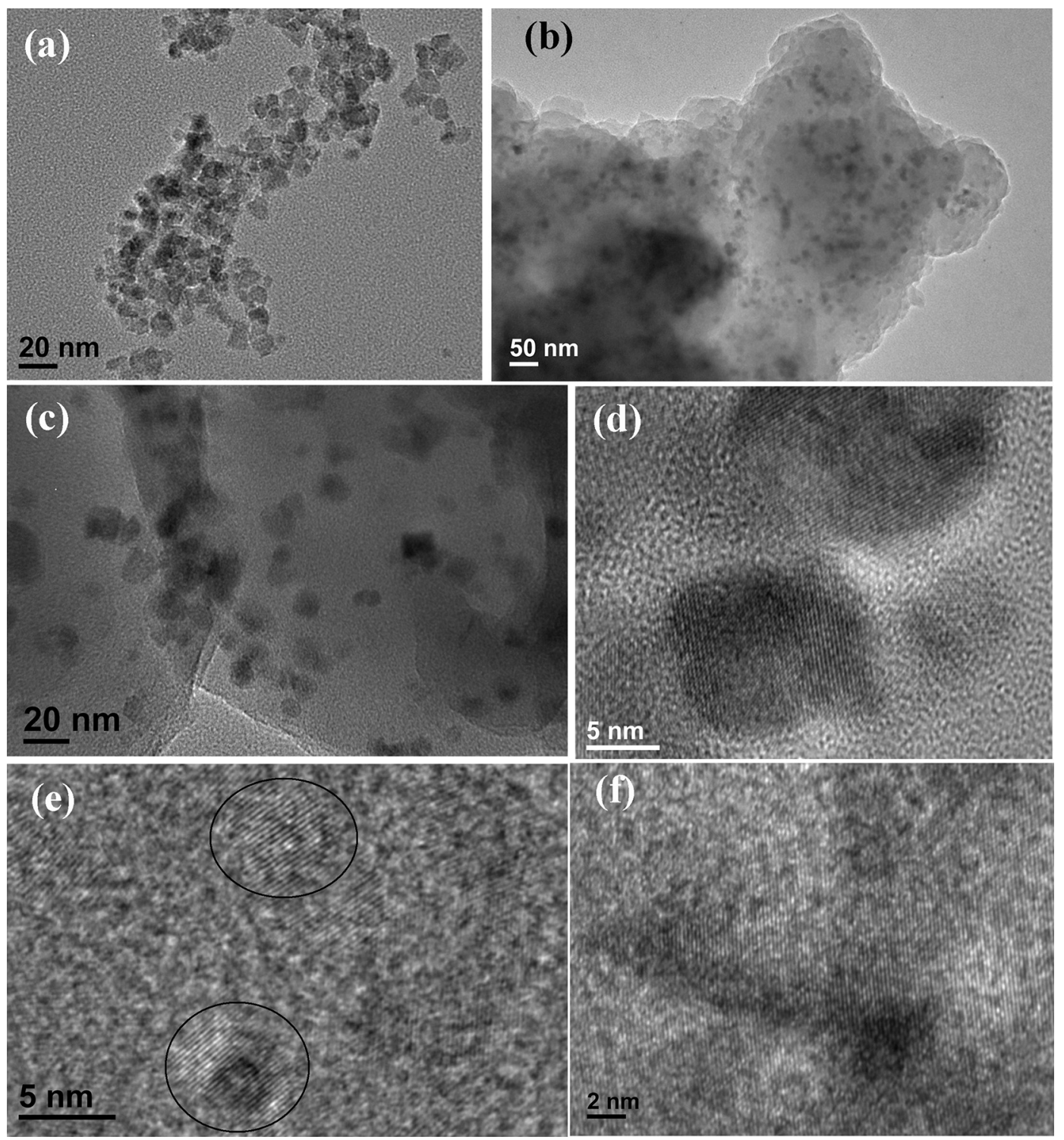

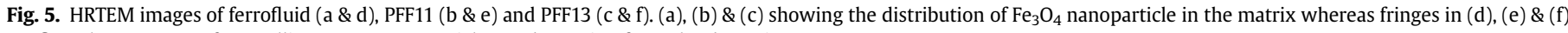
confirm the presence of crystalline $\mathrm{Fe}_{3} \mathrm{O}_{4}$ nanoparticles as determine from the d-spacing.

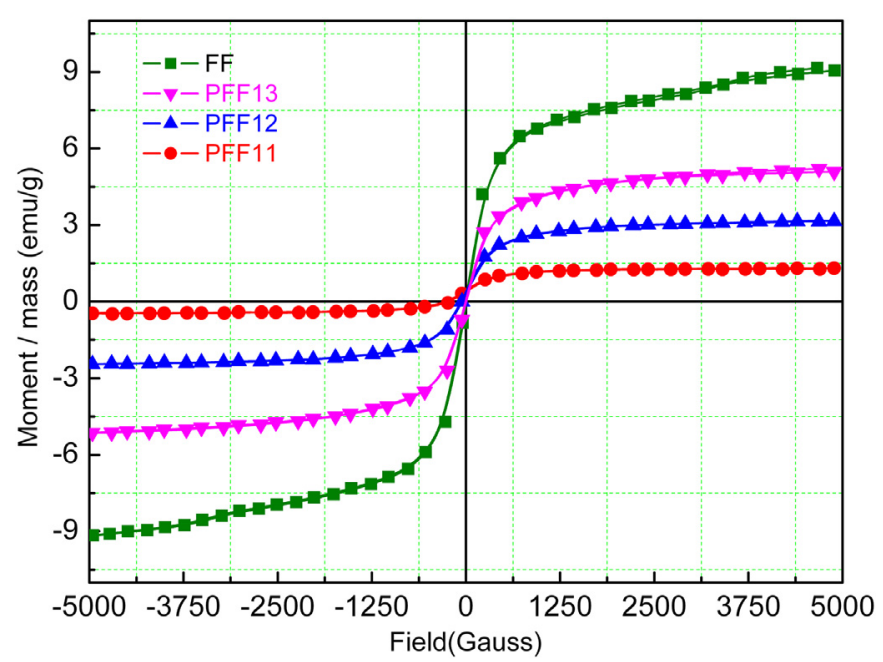

Fig. 6. The change in magnetic moment per unit mass with applied field for FF, PFF11, PFF12 and PFF13 nanocomposites. increasing the volume fraction ratio of $\mathrm{FF}$ in the composite the magnetization value increases from 1.3 to $5.5 \mathrm{emu}^{-1}$ for PFF13 composite at an external field of $5 \mathrm{kOe}$. These results reveal the ferromagnetic nature of the PFF composites. The $M_{\mathrm{s}}$ value increases due to the high poly-dispersivity of the ferrofluid in the polymer matrix. The surface area, the number of dangling bonded atoms and unsaturated co-ordination on the surface of polymer matrix are all enhanced.

\subsection{Electromagnetic interference shielding investigations}

From the interaction of electromagnetic wave with the shielding material, electrons and other charged particles produce a scattered or induced field inside the material in response to the incident wave. The total field is affected by this induced field inside the material and modifies the charge motion [28,29]. A portion of the wave is reflected back through interaction with surface charges when an incident wave strikes the shielding material. Therefore, the material used as the EMI shield tends to be conductive, due to 
mobile charge carriers. The EMI shielding effectiveness (SE) of a material can be expressed as [30]

$\mathrm{SE}(\mathrm{dB})=-10 \log \frac{P_{\mathrm{T}}}{P_{\mathrm{I}}}=\mathrm{SE}_{\mathrm{R}}+\mathrm{SE}_{\mathrm{A}}+\mathrm{SE}_{\mathrm{M}}$

where, $P_{\mathrm{T}}$ and $P_{\mathrm{I}}$ are the transmitted and incident electromagnetic power, whereas $\mathrm{SE}_{\mathrm{R}}, \mathrm{SE}_{\mathrm{A}}$ and $\mathrm{SE}_{\mathrm{M}}$ are the shielding effectiveness due to reflection, absorption and multiple reflections, respectively. The terms in Eq. (2) can be described as $\mathrm{SE}_{\mathrm{R}}=-10 \log (1-R)$ and $\mathrm{SE}_{\mathrm{A}}=-10 \log [T /(1-R)]$ while $\mathrm{SE}_{\mathrm{M}}$ is negligible when $\mathrm{SE}>10 \mathrm{~dB}$ [31]. Fig. 7a shows the variation of the $\mathrm{SE}_{\mathrm{A}}$ and $\mathrm{SE}_{\mathrm{R}}$ with frequency for PPy and PFF composites in 12.4-18.0 GHz (Ku-band). From the figure, it has been revealed that PFF composites show absorption dominated SE rather than reflection. In polypyrrole composite sheets with matching thickness of $\sim 2.0 \mathrm{~mm}$, maximum SE due to absorption $\left(\mathrm{SE}_{\mathrm{A}(\mathrm{max})}\right)$ has been ca. 20.4, 18.6 and $18.1 \mathrm{~dB}$ for PFF13, PFF12, and PFF11 samples at $18.0 \mathrm{GHz}$, respectively whereas the $\mathrm{SE}_{\mathrm{A}(\max )}$ value of $17.6 \mathrm{~dB}$ has been observed for PPy. For the reflection part, $\mathrm{SE}_{\mathrm{R}}$ has been ca. 3.1, 2.9, 2.5 and $5.2 \mathrm{~dB}$ for PFF13, PFF12, PFF11 and PPy samples at $18.0 \mathrm{GHz}$, respectively. The results demonstrate that the absorption of microwave and overall shielding effectiveness $\left(\mathrm{SE}=\mathrm{SE}_{\mathrm{A}}+\mathrm{SE}_{\mathrm{R}}\right)$ up to $23.5 \mathrm{~dB}$ ( 99.5\% attenuation) for PFF13, increases with the concentration of the ferrofluid in the composite. The increase in the absorption with the addition
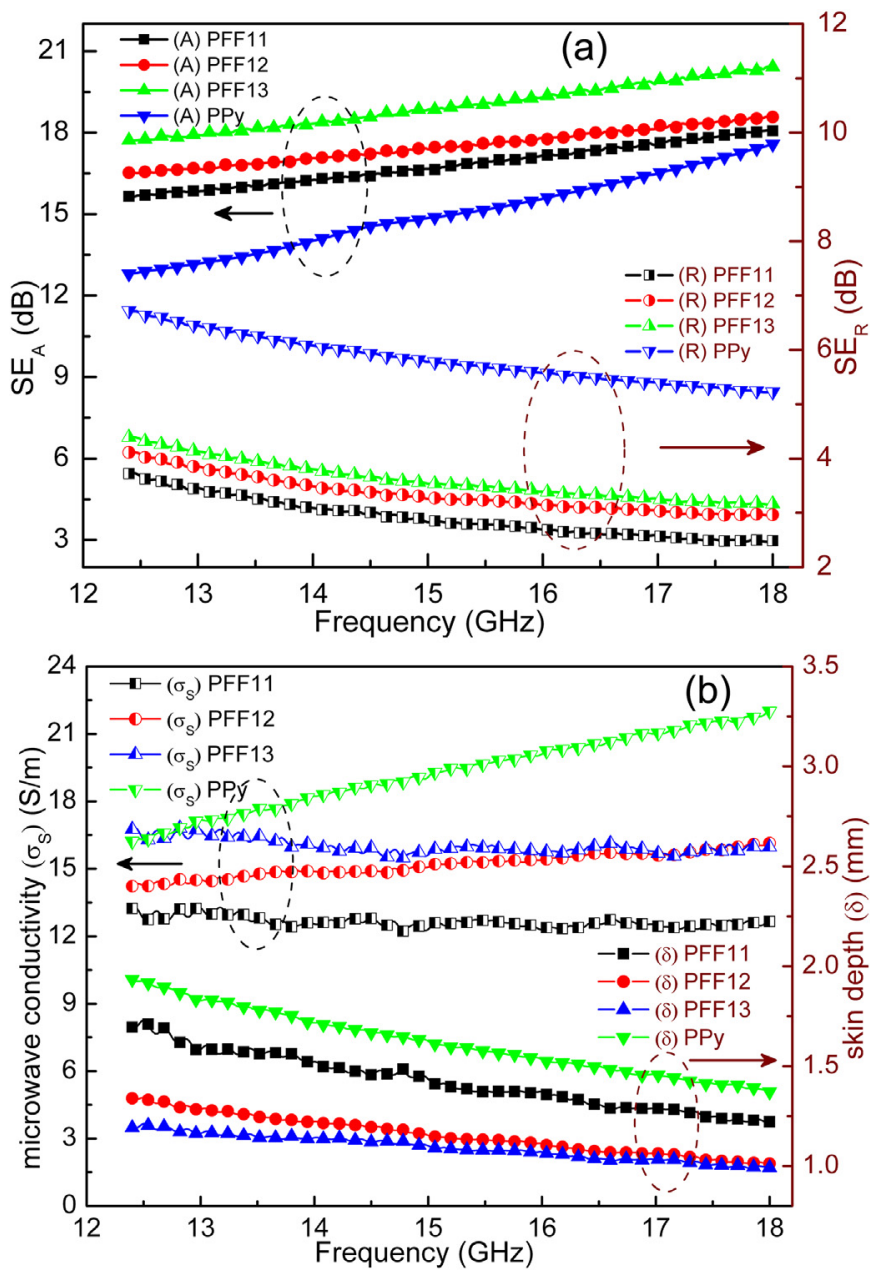

Fig. 7. (a) Dependence of shielding effectiveness $\left(\mathrm{SE}_{\mathrm{A}}\right.$ and $\left.\mathrm{SE}_{\mathrm{R}}\right)$ of PPy and PFF composites as a function of frequency, showing the effect of ferrofluid concentration on the SE value of the nanocomposites for sample thickness $d \sim 2.0 \mathrm{~mm}$. (b) Variation of microwave conductivity and skin depth of PPy and PFF composites as a function of frequency. of ferrofluid in PFF samples may be attributed to the higher dielectric and magnetic losses induced by $\mathrm{Fe}_{3} \mathrm{O}_{4}$ nanoparticles present in ferrofluid. In case of PPy, absorption is still the dominating factor but the reflection part is on the higher side, which makes it less favorable as microwave absorbing material.

For an electrically thick sample, dependence of SE on dielectric properties and magnetic properties can be expressed as [32,33]

$$
\begin{aligned}
& \mathrm{SE}_{\mathrm{A}}(\mathrm{dB})=20 \frac{d}{\delta} \log e=20 d \sqrt{\frac{\mu_{\mathrm{r}} \omega \sigma_{\mathrm{S}}}{2}} \cdot \log e \\
& \mathrm{SE}_{\mathrm{R}}(\mathrm{dB})=10 \log \left(\frac{\sigma_{\mathrm{S}}}{16 \omega \mu_{\mathrm{r}} \varepsilon_{\mathrm{o}}}\right)
\end{aligned}
$$

where, $d$ is the thickness of the shield, $\mu_{\mathrm{r}}$ is the magnetic permeability, $\delta=\sqrt{2 / \mu_{\mathrm{r}} \omega \sigma_{\mathrm{S}}}$ is the skin depth, $\sigma_{\mathrm{S}}=\omega \varepsilon_{0} \varepsilon^{\prime \prime}$ is the frequency dependent microwave conductivity [30], $\varepsilon^{\prime \prime}$ is the imaginary part of permittivity (dielectric loss factor), $\omega$ is the angular frequency ( $\omega=2 \pi f$ ), and $\varepsilon_{0}$ is the permittivity of the free space. From Eqs. (3) and (4) it is observed that absorption of microwave increases while the reflection part decreases with the frequency. The absorption loss, $\mathrm{SE}_{\mathrm{A}}$, is a function of the product $\sigma_{\mathrm{s}} \mu_{\mathrm{r}}$ and is caused by the loss of heat under the action between electric dipole and/or magnetic dipole in the material and the electromagnetic field. The dielectric loss mainly depends on the conductivity of materials whereas the magnetic loss not only results from electrical conductivity but also magnetic permeability [34]. Therefore, magnetic lossy materials have typical high absorption efficiency as compared to that of dielectric loss materials. For the reflection part, the conducting particles in the composites interact with the electro-magnetic field that leads to the reflection loss $\left(\mathrm{SE}_{\mathrm{R}}\right)$ which depends on the ratio $\sigma_{\mathrm{S}} /$ $\mu_{\mathrm{r}}$. Therefore, materials with large conductivity and small magnetic permeability show a higher reflection loss. Fig. 7b demonstrates the variation of microwave conductivity $\left(\sigma_{\mathrm{S}}=\omega \varepsilon_{0} \varepsilon^{\prime \prime}\right)$ and skin depth $\left(\delta=\sqrt{2 / \mu_{\mathrm{r}} \omega \sigma_{\mathrm{S}}}\right)$ with frequency as calculated from the dielectric data. It is observed that the microwave conductivity and the skin depth almost remain constant in the measure frequency range. Higher value $\sigma_{\mathrm{s}}$ has been observed for the PPy due to higher dielectric loss as compared to PFF composites but PFF composites are more prone to the absorption because of the combine effect of dielectric and magnetic loss. In addition, dc conductivity decrease with the increase in the concentration of ferrofluid because $\mathrm{Fe}_{3} \mathrm{O}_{4}$ being ferrite having conductivity of the order of $10^{-10} \mathrm{~S} \mathrm{~cm}^{-1}$ try to hinder the conduction of the electron through the polypyrrole chain. But, due to the unique core shell type the decrease in the conductivity is very less as compared to the polypyrrole. On the other, when we discuss about the microwave conductivity i.e. $\sigma_{\mathrm{S}}=\omega \varepsilon_{0} \varepsilon^{\prime \prime}$, it depends on the dielectric loss in the composites which intern depends on the dissipation of energy and polarization. But here also the change in the microwave conductivity is small. In the present case for PFF13 sample, dc conductivity of $0.05 \mathrm{~S} \mathrm{~cm}^{-1}$ and ac conductivity of $\sim 16.5 \mathrm{~S} \mathrm{~cm}^{-1}$ has been recorded for the composite sheet. Now, using Maxwell's equations, which relates the applied electric field with displacement field, it can be shown that $\varepsilon^{\prime \prime}$ represents the losses in the system of bound charges exactly in the same way as the free charges are responsible for conductivity losses. In analogy with dc conductivity, we can identify $\omega \varepsilon_{0} \varepsilon^{\prime \prime}$ as the conductivity of the sample under an applied ac electric field and the ac conductivity from which the $\varepsilon^{\prime \prime}$ can be defined as

$$
\varepsilon^{\prime \prime}=\frac{\sigma_{\mathrm{S}}}{\omega \varepsilon_{0}}
$$

It is important to mention here that the ac conductivity has been defined as the losses due to bound charges; hence, as such there 
should be no losses under dc electric field. However, every material will have some free charge carriers and under the applied low frequency or even at microwave frequencies, these charge carriers can follow the field and cause conduction current giving rise to energy loss. Under a dc electric field, we have contribution from free charge carriers only; however, in the ac case, the energy loss will be both due to free and bound charge carriers [35,36]. Hence, the total $\varepsilon^{\prime \prime}$ in terms of conductivity can be expressed as [37,38]

$\varepsilon^{\prime \prime}=\frac{\sigma_{\mathrm{dc}}}{\omega \varepsilon_{0}}+\frac{\sigma_{\mathrm{AC}}}{\omega \varepsilon_{\mathrm{o}}}$

To further analyze the shielding mechanism, complex permittivity $\left(\varepsilon_{\mathrm{r}}=\varepsilon^{\prime}-j \varepsilon^{\prime \prime}\right)$ and permeability $\left(\mu_{\mathrm{r}}=\mu^{\prime}-j \mu^{\prime \prime}\right)$ have been calculated using scattering parameters $\left(S_{11} \& S_{21}\right)$ based on the calculations given in Nicholson, Ross and Weir method $[39,40]$ and demonstrated in Fig. 8. The real part $\left(\varepsilon^{\prime}\right)$ is mainly associated with the amount of polarization occurring in the material and the imaginary part $\left(\varepsilon^{\prime \prime}\right)$ is related to the dissipation of energy (dielectric loss) [41]. Similarly, real part $\left(\mu^{\prime}\right)$ is related to the magnetic field induced in the material and the imaginary part $\left(\mu^{\prime \prime}\right)$ represents magnetic loss. The dielectric properties of the material results from ionic, electronic, orientational and space charge polarization. In composite systems, due to the heterogeneity of the material, the contribution to the space charge polarization dominates. While in conjugated polymers space charge polarization appears due to a
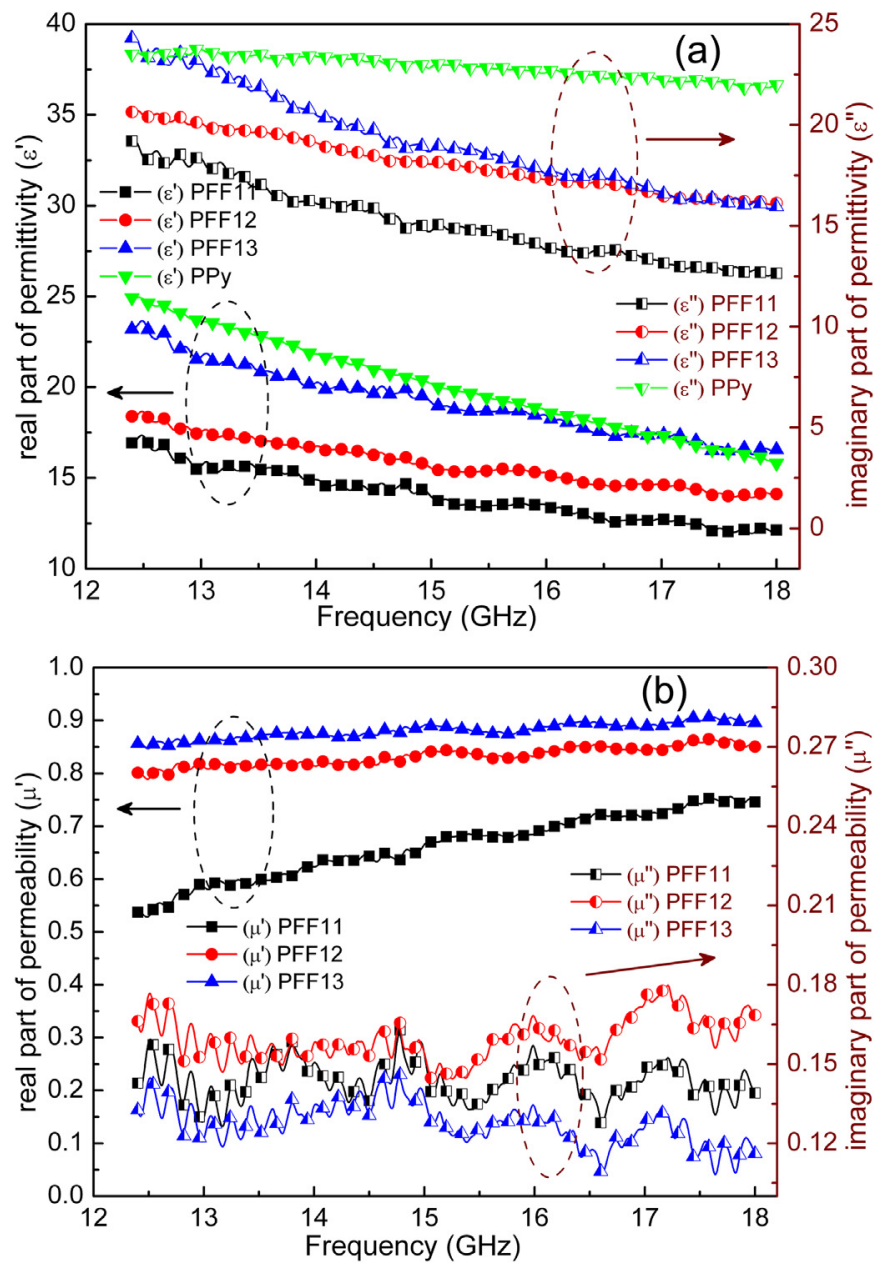

Fig. 8. Behavior of (a) real $\left(\varepsilon^{\prime}\right)$ and imaginary $\left(\varepsilon^{\prime \prime}\right)$ part of complex permittivity of PPy and PFF composites as a function of frequency. Dependence of real $\left(\mu^{\prime}\right)$ and imaginary $\left(\mu^{\prime \prime}\right)$ part of magnetic permeability of PFF composites on frequency. polaron/bipolaron system that is mobile and free to move along the chain and orientational polarization due to bound charges (dipoles) which have only restricted mobility. Fig. 8a reveals that $\varepsilon^{\prime} \& \varepsilon^{\prime \prime}$ decreases with the increase in frequency of the applied field because the dipoles present in the system cannot reorient themselves fast enough to respond to applied electric field. In PFF13 incorporation of ferrofluid in the conducting polypyrrole matrix leads to higher values of dielectric constant $\left(\varepsilon^{\prime}=23.1\right)$ and dielectric loss $\left(\varepsilon^{\prime \prime}=24.3\right)$. This arises due to the difference in the relative dielectric constant of ferrofluid and the polypyrrole that results in the accumulation of more space charges and strong orientational polarization that consequently leads to the improved values of microwave absorption. The magnetic losses are induced due to the presence of magnetic $\mathrm{Fe}_{3} \mathrm{O}_{4}$ nanoparticles in the ferrofluid. The variation in the real $\left(\mu^{\prime}\right)$ and imaginary $\left(\mu^{\prime \prime}\right)$ part of permeability with frequency is shown in Fig. 8b. Both real and imaginary parts of the permeability remain constant with a little fluctuation in the measured frequency range. The magnetic loss express by the imaginary part of complex permeability, mainly occur because of magnetic hysteresis and domain-wall displacement in the material. In $\mathrm{Fe}_{3} \mathrm{O}_{4}$, due to the large anisotropy field, the coupling of the magnetic dipole is strong. When the frequency of the applied field increases the magnetic dipole try to rotate with the frequency but at higher frequencies due to strong anisotropy, the induced magnetization $(M)$ lags behind the applied field $(H)$ which results in magnetic losses. The larger is the anisotropy, the higher is the difference in $M$ and $H$ and more are the magnetic losses occurring in the material. In magnetic nanoparticles, the rotation of domains becomes difficult due to the effective anisotropy (magneto-crystalline anisotropy and shape anisotropy). The effective anisotropy coefficient can enhance significantly with decreasing particle size due to the surface effect and microstructure defects $[42,43]$. The dielectric and magnetic loss in the whole frequency range proves the balanced property of EM matching in the composite suggesting that the enhanced microwave absorption properties unambiguously result from the cooperative effect of polypyrrole and ferrofluid.

\section{Conclusions}

In conclusion, aqueous ferrofluid has been successfully incorporated in the polypyrrole matrix by in situ chemical oxidative polymerization results in the coral type morphology for PFF composites. The incorporation of ferrofluid leads to light weighted nanoarchitectured composite having improved microwave absorption properties due to better interfacial dipolar polarization and higher anisotropic energy. PFF nanocomposite demonstrates strong microwave absorption properties in $12.4-18 \mathrm{GHz}$ having $\mathrm{SE}_{\mathrm{A}}$ value of $20.4 \mathrm{~dB}$ with nominal reflection loss of $3.1 \mathrm{~dB}$. The high absorption properties of PFF composites mainly arise from the high dielectric and magnetic losses. Thus, ferrofluid based polypyrrole nanoarchitectured composite with moderate conductivity and magnetization can be used in a number of commercial applications in electronic devices.

\section{Acknowledgments}

Authors wish to thank Director N.P.L \& Director DITE for their keen interest in the work. The authors also thank Dr. N. Vijayan for recording XRD data and Dr. R. K. Kotnala for VSM measurements of our samples.

\section{References}

[1] J.M. Thomassin, C. Pagnoulle, L. Bednarz, I. Huynen, R. Jerome, C. Detrembleur, J. Mater. Chem. 18 (2008) 792-796. 
S. Varshney et al. / Materials Chemistry and Physics 143 (2014) 806-813

813

[2] V.K. Singh, A. Shukla, M.K. Patra, L. Saini, R.K. Jani, S.R. Vadera, N. Kumar, Carbon 50 (2012) 2202-2208.

[3] G. Wang, Z. Gao, S. Tang, C. Chen, F. Duan, S. Zha, S. Lin, Y. Feng, L. Zhou, Y. Qin, ACS Nano 6 (2012) 11009-11017.

[4] T. Chen, F. Deng, J. Zhu, C. Chen, G. Sun, S. Maa, X. Yang, J. Mater. Chem. 22 (2012) 15190-15197.

[5] D.X. Yan, P.G. Ren, H. Pang, Q. Fu, M.B. Yang, Z.M. Li, J. Mater. Chem. 22 (2012) $18772-18774$

[6] H.R. Kim, K. Fujimori, B.S. Kim, I.S. Kim, Compos. Sci. Technol. 72 (2012) $1233-$ 1239.

[7] H.R. Kim, B.S. Kim, I.S. Kim, Mater. Chem. Phys. 135 (2012) 1024-1029.

[8] X. Gu, W. Zhu, C. Jia, R. Zhao, W. Schmidt, Y. Wang, Chem. Commun. 47 (2011) $5337-5339$

[9] F. Xia, J. Liu, D. Gu, P. Zhao, J. Zhang, R. Che, Nanoscale 3 (2011) 3860.

[10] N. Li, Y. Huang, F. Du, X. He, X. Lin, H. Gao, Y. Ma, F. Li, Y. Chen, P.C. Eklund, Nano Lett. 6 (2006) 1141-1145.

[11] M. Wan, W. Li, J. Polym. Sci. Part A: Polym. Chem. 35 (1997) 2129-2136.

[12] T.T. Tung, J.F. Feller, T.Y. Kim, H. Kim, W.S. Yang, K.S. Suh, J. Polym. Sci. Part A: Polym. Chem. 50 (2012) 927-935.

[13] K. Chen, C. Xiang, L. Li, H. Qian, Q. Xiao, F. Xu, J. Mater. Chem. 22 (2012) 64496455.

[14] W. Wang, S.P. Gumfekar, Q. Jiao, B. Zhao, J. Mater. Chem. C 1 (2013) 28512859.

[15] Y. Xie, X. Hong, Y. Gao, M. Li, J. Liu, J. Wang, J. Lu, Synth. Met. 162 (2012) $677-681$.

[16] L. Yang, M.C. Gupta, K.L. Dudley, R.W. Lawrence, Nano Lett. 5 (2005) 2131 2134.

[17] A.G. Roca, J.F. Marco, M.P. Morales, C.J. Serna, J. Phys. Chem. C 111 (2007) 18577-18584.

[18] M.P. Morales, S.V. Verdaguer, M.I. Montero, C.J. Serna, A. Roig, L.I. Casas, B. Martınez, F. Sandiumenge, Chem. Mater. 11 (1999) 3058-3064.

[19] K. Singh, A. Ohlan, V.H. Pham, R. Balasubramaniyan, S. Varshney, J. Jang, S.H. Hur, W.M. Choi, M. Kumar, S.K. Dhawan, B.S. Kong, J.S. Chung, Nanoscale 5 (2013) 2411-2420.

[20] C. Cui, Y. Du, T. Li, X. Zheng, X. Wang, X. Han, P. Xu, J. Phys. Chem. B 116 (2012) 9523-9531.
[21] M.S. Cao, J. Yang, W.L. Song, D.Q. Zhang, B. Wen, H.B. Jin, Z.L. Hou, J. Yuan, ACS Appl. Mater. Interfaces 4 (2012) 6949-6956.

[22] K. Singh, A. Ohlan, A.K. Bakhshi, S.K. Dhawan, Mater. Chem. Phys. 119 (2010) 201-207.

[23] A. Ohlan, K. Singh, A. Chandra, S.K. Dhawan, ACS Appl. Mater. Interfaces 2 (2010) 927-933.

[24] J. Jiang, L.H. Ai, J.L. Zheng, J. Macromol. Sci. Part B: Phys. 49 (2010) 652-657.

[25] I. Seo, M. Pyo, G. Cho, Langmuir 18 (2002) 7253-7257.

[26] M. Wan, Adv. Mater. 20 (2008) 2926-2932.

[27] H. Yang, W. Jiang, Y. Lu, Mater. Lett. 61 (2007) 2789-2793.

[28] C.R. Paul, Introduction to Electromagnetic Compatibility, second ed., John Wiley and Sons, New Jersey, 2006.

[29] K.L. Kaiser, Electromagnetic Shielding, CRC Press, Boca Raton, FL, 2006.

[30] A. Ohlan, K. Singh, A. Chandra, S.K. Dhawan, Appl. Phys. Lett. 93 (2008) 053114.

[31] S. Varshney, K. Singh, A. Ohlan, V.K. Jain, V.P. Dutta, S.K. Dhawan, J. Alloys Compd. 538 (2012) 107-114.

[32] N. Gandhi, K. Singh, A. Ohlan, D.P. Singh, S.K. Dhawan, Compos. Sci. Technol. 71 (2011) 1754-1760.

[33] N.F. Colaneri, L.W. Shacklette, IEEE Trans. Instrum. Meas. 41 (1992) 291-297.

[34] M. Wan, Conducting Polymers with Micro or Nanometer Structure, Springer, Berlin, Heidelberg, New York, 2008.

[35] R. Singh, J. Kumar, R.K. Singh, R. C Rastogi, V. Kumar, New J. Phys. 9 (2007) 40.

[36] S. Varshney, A. Ohlan, K. Singh, V.K. Jain, V.P. Dutta, S.K. Dhawan, Sci. Adv. Mater. 5 (2013) 881-890.

[37] N.F. Mott, E.A. Davis, Electronic Processes in Non-crystalline Materials, Oxford University Press, London, 1979.

[38] R. Singh, J. Kumar, R.K. Singh, A. Kaur, R.D.P. Sinha, N.P. Gupta, Polymer 47 (2006) 5919-5928.

[39] A.M. Nicolson, G.F. Ross, IEEE Trans. Instrum. Meas. 19 (1970) 377-382.

[40] W.B. Weir, Proc. IEEE 62 (1974) 33-36.

[41] D.S.P. Devi, P.K. Bipinbal, T. Jabin, S.K.N. Kutty, Mater. Des. 43 (2013) 337-347.

[42] D.A. Dimitrov, G.M. Wysin, Phys. Rev. B 51 (1995) 11947-11950.

[43] V.P. Shilov, J.C. Bacri, F. Gazeau, F. Gendron, R. Perzynski, Y.L. Raikher, J. Appl. Phys. 85 (1999) 6642-6647. 